\title{
Idiopathic Spontaneous Intramedullary Hemorrhage: A Report of a Rare Case of Repeated Intramedullary Hemorrhage with Unknown Etiology
}

\author{
Byeong Sam Choi, Sungjoon Lee \\ Department of Neurosurgery, Inje University Haeundae Paik Hospital, Inje University College of Medicine, Busan, Korea
}

A 48-year-old woman presented with acute voiding difficulty, numbness and weakness of both lower extremities. Magnetic resonance imaging (MRI) showed an intramedullary hemorrhagic mass that extended from T9 to T10. T8-T10 laminotomy and surgical removal of the hemorrhagic mass was performed. The pathological diagnosis was hematoma. Her neurological status remained the same after the operation. At 5 days post-operation, the patient suddenly became paraplegic, and MRI that was immediately performed revealed a recurrent intramedullary hemorrhage. Emergent surgical evacuation was performed. Again, histological examination showed only hematoma, without any evidence of abnormal vessels or a tumor. A postoperative MRI revealed no abnormal lesions other than those resulting from postoperative changes. At a 9-month follow up, the patient could walk a short distance with the aid of a walker. Because spontaneous intramedullary hemorrhage with unknown etiology is very rare, it is essential to perform a meticulous inspection of the hemorrhagic site to find the underlying cause. Repeated hemorrhage can occur; therefore, close observation of patients after surgery is important in cases without an apparent etiology. Urgent surgical evacuation is important to improve outcomes in these cases.

Key Words: Intramedullary hemorrhage $\cdot$ Intraspinal hemorrhage $\cdot$ Hematomyelia $\cdot$ Spinal cord $\cdot$ Idiopathic $\cdot$ Spontaneous

\section{INTRODUCTION}

Spontaneous intramedullary hemorrhage is a rare disease entity, and most cases have specific etiologies, such as vascular malformations and tumorous lesions, ${ }^{6,11,12)}$. There are also reports indicating that anticoagulation therapy and hemophilia cause spontaneous intramedullary hemorrhage ${ }^{1,3,13)}$. It is extremely rare for this to occur without an apparent underlying etiology, and only a few such cases have been reported ${ }^{4,6,8-10)}$.

Recurrent hemorrhage is thought to be the cause of progressive and stepwise neurological deterioration in vascular malformations of the spinal $\operatorname{cord}^{2}$, and a high risk of rebleeding from a vascular malformation is the reason why total excision of the pathology is important. Although there have been some reports suggesting the occurrence of recurrent bleeding in chronic forms of intramedullary hemorrhage without apparent

- Received: September 8, 2015 • Revised: November 5, 2015

- Accepted: November 10, 2015

Corresponding Author: Sungjoon Lee, MD

Department of Neurosurgery, Inje University Haeundae Paik Hospital, Inje University College of Medicine, 875 Haeundae-ro, Haeundae-gu, Busan 48108, Korea

Tel: +82-51-797-0241, Fax: +82-51-797-0841

E-mail: potata98@naver.com

@This is an Open Access article distributed under the terms of the Creative Commons Attribution Non-Commercial License (http://creativecommons.org/ licenses/by-nc/3.0/) which permits unrestricted non-commercial use, distribution, and reproduction in any medium, provided the original work is properly cited. etiology ${ }^{7,10)}$, it is extremely rare for recurrent intramedullary hemorrhage to occur acutely without a specific cause.

Here, we present a case of recurrent idiopathic spontaneous intramedullary hemorrhage. While we describe this rare event, we discuss its etiology, and the diagnostic, therapeutic and prognostic implications of this disease including a review of the relevant literature.

\section{CASE REPORT}

A 48-year-old woman visited our emergency department complaining of voiding difficulty, weakness and sensory disturbances in both lower extremities, which had started 7 days ago prior to her visit. An initial neurological examination revealed grade III motor power for the right and grade IV motor power for left lower extremity, and she was unable to walk. Sensation was decreased below the T10 dermatome. Her anal tone was decreased, and voiding was not possible. A trauma history was denied. She had been taking an antihypertensive medication for 4 years. Otherwise she had no known diseases such as blood dyscrasias, bleeding disorders, cardiovascular disorders, and various other central nervous system disorders. Laboratory tests showed no abnormal findings. Thoraco-lumbar spine magnetic resonance imaging (MRI) revealed an intramedullary mass lesion at the T9-10 level, 
which showed as a high signal in a T1 weighted image (Fig. 1A) and a low signal in a T2 weighted image (Fig. 1B). Following gadolinium injection, subtle enhancement at the periphery of the mass was observed (Fig. 1C, 1D). Our preoperative diagnosis was hemorrhagic intramedullary ependymoma.

A T8-T10 laminotomy was performed. On inspection of the spinal cord, no abnormal findings, such as abnormal vessels or discoloration, were found. Midline myelotomy along the dorsal columns was performed, and a dark-colored hematoma was evacuated. It was mostly liquefied, but some of it was not. Keeping cavernous malformation in mind as a possible diagnosis, the dark blood clots were carefully dissected from the spinal cord and sent for histopathological examination. On careful inspection of the intraspinal cavity, neither abnormal vessels nor tumorous lesions were observed. The hematoma was near totally removed, and meticulous bleeding control was followed. Postoperative MRI revealed most of the lesion was removed, and no abnormal findings other than postoperative changes were observed (Fig. 2). The pathology report confirmed only hematoma. After operation, motor and sensory
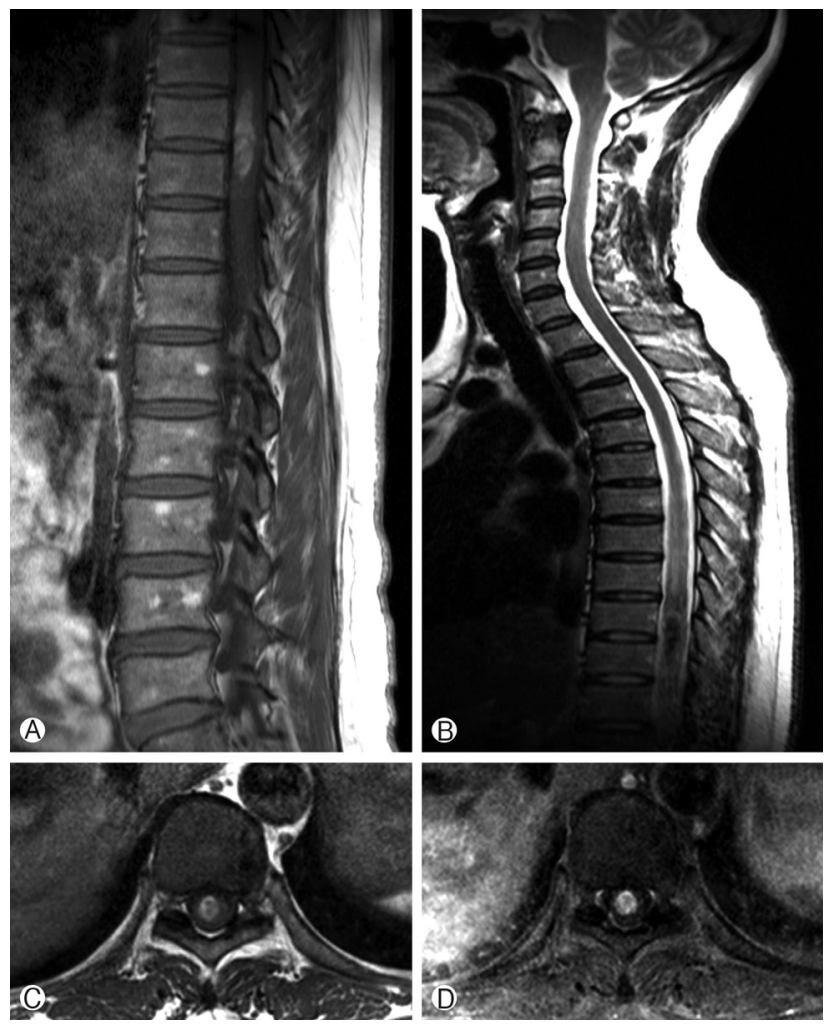

Fig. 1. Preoperative MRI revealed an intramedullary mass lesion from the T9 to T10 level. A T1 weighted sagittal image showing high signal intensity (A). A T2 weighted image showing low signal intensity (B). Compared to a non-enhanced $\mathrm{T} 1$ weighted axial image (C), subtle peripheral enhancement was observed in the enhanced $\mathrm{T} 1$ weighted axial image (D). functions were nearly the same as preoperative status. On the second postoperative day, voiding function was recovered.

On the fifth postoperative day, the patient became suddenly paraplegic. Motor power in both legs was grade 0-1. Only the left ankle and toe showed grade 2 movement. An MRI was immediately taken, and it showed an increased extent of intramedullary hemorrhage at the operation site (Fig. 3). The blood pressure was 130/90 mmHg. Laboratory examination revealed no abnormality of coagulation profile (prothrombin time international normalized ratio, 0.99 (reference 0.851.3), activated partial thromboplastin time, 30.7 seconds (refe-

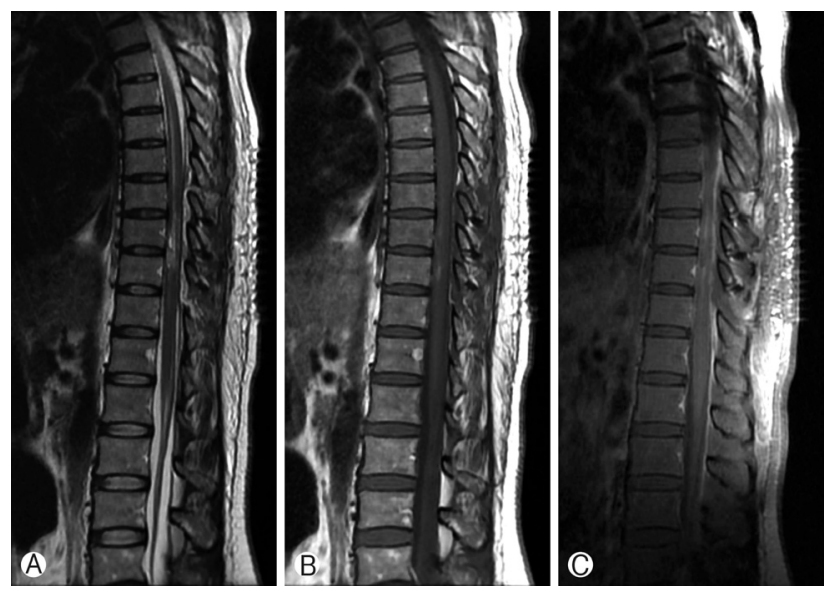

Fig. 2. A postoperative MRI taken 2 days after the first surgery. No abnormal signal voiding or enhanced lesions, other than postoperative changes, were observed. (A) T2 weighted sagittal image. (B) Non-enhanced T1 weighted sagittal image. (C) Enhanced $\mathrm{Tl}$ weighted sagittal image.

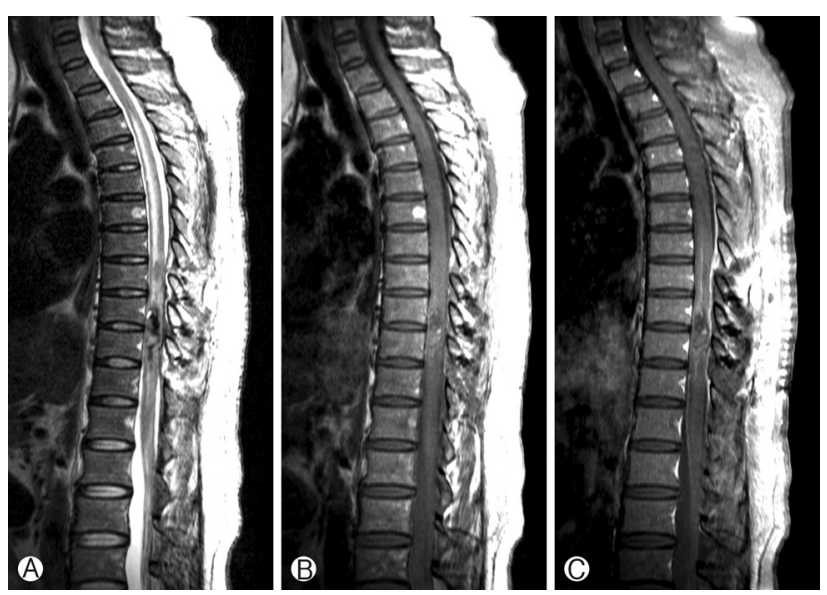

Fig. 3. Sudden weakness in both of the lower extremities occurred at postoperative day 5 after the first surgery. An immediate T-spine MRI was performed. An intramedullary hemorrhage at the operation site had newly appeared. The hemorrhage showed low signal intensity in a T2 weighted sagittal image (A) and high signal intensity in a $\mathrm{Tl}$ weighted image (B) without enhancement (C). 


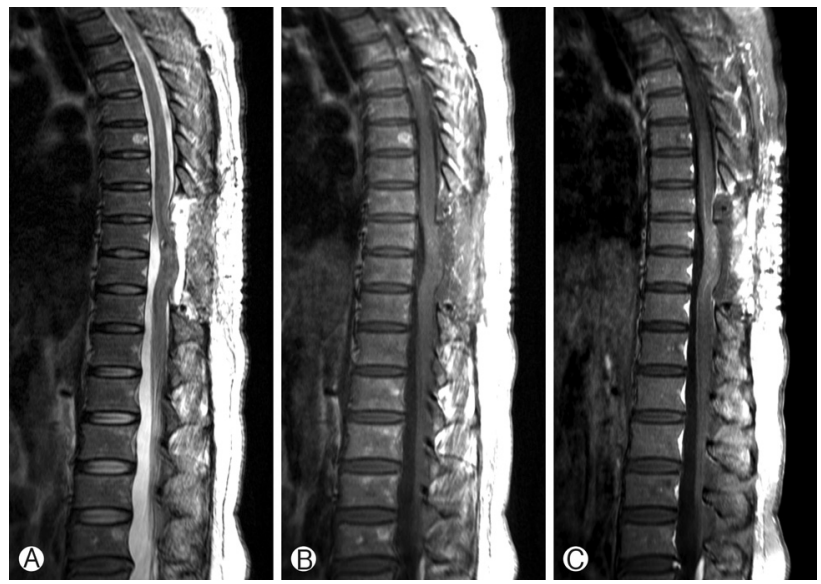

Fig. 4. A postoperative MRI taken a week after the second surgery. A state of total laminectomy from T8 to T10 and focal cerebrospinal fluid collection at the operation site were observed. No abnormal lesions of the spinal cord, other than normal postoperative changes, were observed. (A) T2 weighted sagittal image. (B) Non-enhanced T1 weighted sagittal image. (C) Enhanced T1 weighted sagittal image.

rence 30-47 seconds)). Emergent operation for T8-T10 laminectomy and hematoma evacuation was performed. Again, the hematoma cavity was carefully inspected. However, we failed to identify any abnormal vasculature or tumorous lesion that might have caused rebleeding. Lesions which might have caused the recurrent bleeding were dissected and biopsied for pathologic diagnosis. During the operation, the spinal cord was so severely swollen that we had to perform expansile duroplasty using artificial dura. The pathology report again confirmed only hematoma, and no abnormal vessels or atypical cells were identified. A MRI that was taken seven days after the second surgery showed a markedly decreased extent of the intramedullary hemorrhage and a spinal cord herniation to the dorsal side, which was due to the expansile duroplasty. No other pathology was identified (Fig. 4).

The patient was still paraplegic immediately after surgery. A rehabilitation program was arranged, and motor power in both of her lower extremities gradually improved. Three months after surgery, motor power in both right and left legs were improved to grade 2 and 3, respectively. At a 9-month follow-up, she could walk a short distance with the aid of a walker. In addition, self-voiding became possible with a maneuver to increase abdominal pressure.

\section{DISCUSSION}

What makes this case unique from others is that two confirmed events of intramedullary hemorrhage without specific cause occurred, and that the second event happened a few days after the surgery to perform hematoma evacuation with meticulous bleeding control. Evidence for recurrent bleeding in chronic forms of spontaneous intramedullary hemorrhage are present in the literature ${ }^{10)}$, but it was difficult to find a case similar to the present one.

Variable causes of intramedullary hemorrhage, such as vascular malformations, tumorous conditions, and conditions causing coagulopathies and trauma have been proposed ${ }^{4}$. Although it is extremely rare, there are a few reports of cases of intramedullary hemorrhage without an identifiable etiology. MRI is considered the most valuable diagnostic tool ${ }^{4,5)}$. The stages and extent of hematoma can be seen clearly, and sometimes the underlying pathology can be identified. The hematoma usually compresses the surrounding neural tissue and the possibility that small obliterated lesions are present that do not appear on a MRI scan should be considered.

In the present case, the periphery of the hematoma was likely to enhance, and our preoperative diagnosis was intramedullary ependymoma with hemorrhage. However, intraoperative inspection and the pathology report did not find any pathology that might cause hemorrhage. Five days after the initial surgery, rebleeding at the operation site occurred. Because it was possible that the etiology had been missed during the first surgery, possible lesions that might cause the recurrent bleeding were dissected and biopsied during the second surgery. Again, no abnormal lesions other than hematoma were confirmed in the final pathology report. Postoperative MRIs taken after the first and second operations also showed no abnormal enhancement of vessels or mass lesions, except for normal postoperative changes. With these findings, we concluded that the diagnosis of our case was spontaneous idiopathic intramedullary hemorrhage.

The role of conventional angiography is under debate. Some authors disagree with its use if a MRI did not show any abnormal vessels ${ }^{5,6}$. Idiopathic intramedullary hemorrhage is usually diagnosed by surgery when no underlying pathology is found in macro- and microscopy ${ }^{4}$. However, most recurrent hemorrhage cases in central nervous system have a vascular pathology with a bleeding focus, and it is logical to think so in our case. In this context, spinal angiography would have been helpful to identify the etiology of recurrent bleeding, and we think that it was necessary to do the angiography on looking back upon this case.

Most of the literature recommends early diagnosis and surgery for cases of intramedullary hemorrhage with progressive neurologic deterioration ${ }^{46,9)}$. In addition, our case suggests that delayed hemorrhage can occur without the presence of an etiology even after meticulous bleeding control is performed in a previous surgery. Careful surveillance and timely surgery 
seems to be very important. The rebleeding event took place 5 days after the initial surgery in our patient. The motor grade of both lower extremities decreased to $0-1$. Urgent evacuation of the intramedullary hematoma and decompression of the spinal canal was performed. Approximately 9 months later, motor power in both legs improved and the patient could walk a short distance with the aid of a walker. We emphasize that surgical intervention should be performed as soon as possible to preserve and restore neurological functions in cases of idiopathic spontaneous intramedullary hemorrhage.

\section{CONCLUSION}

Because spontaneous intramedullary hemorrhage with unknown etiology is very rare, meticulous inspection of the hemorrhagic site to find the cause is essential. Repeated hemorrhage can occur; close observation of patients after surgery is therefore important in cases without an apparent etiology. Urgent surgical evacuation is important to improve outcomes in such cases.

\section{REFERENCES}

1. Aulakh R, Panigrahi I, Naranje K, Sharda S, Marwaha RK: Spontaneous hematomyelia in a child with hemophilia A: a case report. J Pediatr Hematol Oncol 31:766-767, 2009

2. Barnwell SL, Dowd CF, Davis RL, Edwards MS, Gutin PH, Wilson CB: Cryptic vascular malformations of the spinal cord: diagnosis by magnetic resonance imaging and outcome of sur- gery. J Neurosurg 72:403-407, 1990

3. Brandt M: Spontaneous intramedullary haematoma as a complication of anticoagulant therapy. Acta Neurochir (Wien) 52:7377, 1980

4. Chao CH, Tsai TH, Huang TY, Lee KS, Hwang SL: Idiopathic spontaneous intraspinal intramedullary hemorrhage: a report of two cases and literature review. Clin Neurol Neurosurg 115: 1134-1136, 2013

5. Hwang JH, Sung JK, Hwang SK, Hamm IS, Park YM, Kim SL: Spontaneous hematomyelia: Case report. J Korean Neurosurg Soc 29:411-419, 2000

6. Karavelis A, Foroglou G, Petsanas A, Zarampoukas T: Spinal cord dysfunction caused by non-traumatic hematomyelia. Spinal Cord 34:268-271, 1996

7. Koos WT, Bock F: Spontaneous multiple intramedullary hemorrhages. Case report. J Neurosurg 32:581-584, 1970

8. Kumar S, Kumar Jaiswal A, Singh H: Spontaneous intramedullary hematoma. A case report. J Neurosurg Sci 49:21-23, 2005

9. Licata C, Zoppetti MC, Perini SS, Bazzan A, Gerosa M, Da Pian R: Spontaneous spinal haematomas. Acta Neurochir (Wien) 95:126-130, 1988

10. Matsumura A, Ayuzawa S, Doi M, Enomoto T, Takeuchi S, Yoshii Y, et al: Chronic progressive hematomyelia: case reports and review of the literature. Surg Neurol 51:559-563, 1999

11. Nemoto Y, Inoue Y, Tashiro T, Mochizuki K, Oda J, Kogame $\mathrm{S}$, et al: Intramedullary spinal cord tumors: significance of associated hemorrhage at MR imaging. Radiology 182:793-796, 1992

12. Ogilvy CS, Louis DN, Ojemann RG: Intramedullary cavernous angiomas of the spinal cord: clinical presentation, pathological features, and surgical management. Neurosurgery 31:219-229, 1992

13. Zeidman SM, Olivi A: Cervical intramedullary hemorrhage as a result of anticoagulant therapy. J Spinal Disord 6:456-457, 1993 